\title{
The relationship between intrahepatic portal systemic shunts and microsphere induced portal hypertension in the rat liver
}

\author{
X Li, I S Benjamin, B Alexander
}

\begin{abstract}
Background-Portal hypertension is associated with gross haemodynamic disturbances characterised by high cardiac output, low peripheral vascular resistance, increased splanchnic blood flow, and portal systemic shunting.

Aims-To study the relationship between intrahepatic portal systemic shunts and microsphere induced portal hypertension in the rat liver.
\end{abstract}

Methods-Different sized microspheres were sequentially injected into the portal vein of male Wistar rats.

Results-Steady state portal venous pressure was increased by $102.2(35.6) \%(14.9$ (3.6) $\mathrm{mm} \mathrm{Hg}$ ) and 272.3 (78.0)\% (24.0 (2.2) mm Hg) above the basal pressure following sequential injections of 15 and $80 \mu \mathrm{m}$ diameter microspheres, respectively. Sequential injection of 15,40 , and $80 \mu \mathrm{m}$ diameter microspheres in either ascending or descending order of size did not generate further increases in portal venous pressure. A single injection of $1.8 \times$ $10^{5} 80 \mu \mathrm{m}$ microspheres consistently produced a steady state portal venous pressure of 19.0 (1.3) $\mathrm{mm} \mathrm{Hg}$ but did not approach the much higher value of 36.6 (43.2) $\mathrm{mm} \mathrm{Hg}$ measured during clamping of the portal vein. These data indicate that the opening of patent intrahepatic shunts was responsible for the reduced pressures observed during microsphere injections and further evidence for this was provided by the location of microspheres in the pulmonary vascular bed. The elevation in portal venous pressure achieved by $\mathrm{mi}$ crosphere injections was not significantly different to that produced in rats subjected to partial portal vein ligation $(20.7$ (0.5) $\mathrm{mm} \mathrm{Hg}, p>0.05)$. Wedged hepatic venous pressure decreased from $6.7(0.7)$ to $3.0(0.6) \mathrm{mm} \mathrm{Hg}$ following injection of $80 \mu \mathrm{m}$ microspheres, suggesting a decrease in total hepatic blood flow. Conversely, injection of $15 \mu \mathrm{m}$ microspheres induced an increase in wedged hepatic venous pressure from $7.0(1.0) \mathrm{mm} \mathrm{Hg}$ to $12.4(1.8) \mathrm{mm} \mathrm{Hg}$, indicating a localised redistribution of blood flow at the presinusoidal level of the portal venous vascular network and increased intrahepatic shunt flow.

Conclusion-It is suggested that there may be a protective pathophysiological role for these shunts when the liver is sub- jected to changes which induce acute portal hypertension.

(Gut 1998;42:276-282)

Keywords: portal hypertension; intrahepatic shunts; rat liver; hepatic blood flow

Portal hypertension is associated with gross haemodynamic disturbances characterised by high cardiac output, low peripheral vascular resistance, increased splanchnic blood flow, and portal systemic shunting. ${ }^{1-3}$ Measurements of the portal haemodynamics in man are difficult and usually invasive ${ }^{4-7}$ and therefore reliable experimental animal models are important for research into portal hypertension. A popular model used is the partial portal vein ligation model in the $\mathrm{rat}^{8}$ which induces extrahepatic portal venous hypertension. Carbon tetrachloride and other drug induced models of cirrhosis closely replicate some of the major features of the disease in man, but take a long time to develop and are associated with a high mortality and a wide heterogeneity in the stage and development of cirrhosis. ${ }^{9}{ }^{10}$ Experimental models of secondary biliary cirrhosis induced by bile duct ligation may also exhibit similar limitations. ${ }^{11}$

A new acute model of intrahepatic portal hypertension in the rat induced by intraportal injection of microspheres was reported previously where the existence of intrahepatic portal systemic shunts in the normal rat liver was shown. ${ }^{12}$ Intrahepatic shunts have been reported previously to exist in cirrhotic and to a lesser extent in normal livers in rats ${ }^{13}$ and in man, ${ }^{14}$ where it was suggested that these did not operate under normal conditions. ${ }^{15}$ This hypothesis was partially substantiated by the observation that the maximum elevation of portal venous pressure generated by microsphere injections was only $50 \%$ above the basal value in the initial study. This value was much lower than the increase of approximately $100 \%$ which has been observed in portal vein ligated and cirrhotic models. ${ }^{516}$ Moreover, it was puzzling that the microsphere diameter had little effect on the rise in portal pressure. Two possible explanations were proposed for this: either insufficient numbers of microspheres were injected, and/or opening of intrahepatic portal systemic shunts prevented greater increases.

The aims of the present study were to determine whether: (1) increasing the numbers of microspheres injected would induce further increases in portal venous pressure; (2) injection of 15,40 , and $80 \mu \mathrm{m}$ diameter 
microspheres in either ascending or descending order of diameter affected the portal venous pressure and wedged hepatic venous pressure; (3) injection of microspheres generated greater increases in portal venous pressure compared with the partial portal vein ligated model of portal hypertension; and (4) it was possible to generate a greater degree of portal hypertension than in our previous study by a single injection of a larger number of microspheres or whether the presence of naturally occurring patent, intrahepatic, portal systemic shunts would prevent this.

\section{Materials and methods}

Male Wistar rats (250-350 g) allowed food and water ad libitum, were anaesthetised with fentanyl/fluanisone $(0.3 \mathrm{ml} / \mathrm{kg}$ subcutaneously) and midazolam ( $0.3 \mathrm{ml} / \mathrm{kg}$ subcutaneously). The left carotid artery was cannulated for measurement of mean arterial pressure and following heparinisation $(30 \mathrm{U} / 100 \mathrm{~g}$ intravenously), the abdomen was opened via a midline incision, and the portal vein cannulated via a distal caecal vein for measurement of portal venous pressure and injection of microspheres. To avoid microsphere aggregation a large catheter (outer diameter $1.02 \mathrm{~mm}$ ) was advanced to the main trunk of the portal vein. The splenic pulp pressure was measured through a $23 \mathrm{G}$ butterfly needle carefully inserted into the spleen and provided simultaneous confirmation of measurements of portal venous pressure. Pressures were measured on P23XL (Viggo Spectramed Inc.) pressure transducers which were connected to a Polygraph (P37) recorder (Grass Instruments Inc., USA) for recording of permanent traces. Temperature was maintained at $37(0.5)^{\circ} \mathrm{C}$ by a heating blanket and monitored by a rectal probe (MC 9200 EXACON).

EXPERIMENTAL DESIGN

A total of 38 rats was divided into six groups. Group $1(n=6)$ and group $2(n=6)$ received sequential injections of single sized microspheres of $15 \mu \mathrm{m}$ and $80 \mu \mathrm{m}$ diameter respectively. Group $3(n=6)$ and group $4(n=6)$ received sequential injections of three different sized microspheres in order of increasing diameter $(15+40+80 \mu \mathrm{m}$ and $80+40+15 \mu \mathrm{m}$, respectively, see table 1 ). Two aliquots of 0.2 $\mathrm{ml}$ were injected for each size of microsphere because this (undiluted) volume of microspheres contained the minimum number required to elevate the portal venous pressure to the maximum value obtained for each diameter of microsphere. According to the results of the

Table 1 Intraportally injected aliquots of microspheres

\begin{tabular}{lllll}
\hline Group & Sphere diameter $(\mu \mathrm{m})$ & No of aliquots & No of spheres/aliquot & $\begin{array}{l}\text { Total no of } \\
\text { spheres }\end{array}$ \\
\hline 1 & 15 & 6 & $5.6 \times 10^{6}$ & $3.4 \times 10^{7}$ \\
2 & 80 & 5 & $9.0 \times 10^{4}$ & $4.5 \times 10^{5}$ \\
3 & $15+40+80$ & $15 \mu \mathrm{m}: \times 2$ & $5.6 \times 10^{6}$ & $1.1 \times 10^{7}$ \\
& & $40 \mu \mathrm{m}: \times 2$ & $2.4 \times 10^{5}$ & $4.8 \times 10^{5}$ \\
4 & $80+40+15$ & $80 \mu \mathrm{m}: \times 2$ & $9.0 \times 10^{4}$ & $1.8 \times 10^{5}$ \\
& & $80 \mu \mathrm{m}: \times 2$ & $9.0 \times 10^{4}$ & $1.8 \times 10^{5}$ \\
5 & 80 & $40 \mu \mathrm{m}: \times 2$ & $2.4 \times 10^{5}$ & $4.8 \times 10^{5}$ \\
& & $15 \mu \mathrm{m}: \times 2$ & $5.6 \times 10^{6}$ & $1.1 \times 10^{7}$ \\
& & 2 & $9.0 \times 10^{4}$ & $1.8 \times 10^{5}$ \\
\hline
\end{tabular}

four groups above, two bolus injections of 80 $\mu \mathrm{m}$ microspheres were selected as the suitable dose for the induction of portal hypertension in the model and these were given to group 5 $(n=8)$. Rats in group $6(n=6)$ received saline injections at appropriate time intervals and served as controls. As no significant changes in blood pressure were recorded in this group; on completion of the control protocol, they were also used as acute models of portal hypertension by partial portal vein ligation. At the termination of the experiments, all rats were killed with an overdose of anaesthetic.

INJECTION OF MICROSPHERES

Latex microspheres (Coulter Electronics Ltd, Harpenden, Hertfordshire, UK) were suspended in saline at $37^{\circ} \mathrm{C}$ and numbers per sample injected were counted using a Coulter counter (Model ZB) from a representative sample withdrawn prior to injection. When steady basal pressures had been achieved for at least five minutes, the microspheres were vortexed for 60 seconds before injection. Following withdrawal of a sample for counting from the stock solution, $0.2 \mathrm{ml}$ aliquots were injected via the portal venous catheter over 15-20 seconds and immediately followed by a $0.2 \mathrm{ml}$ saline injection. When a steady portal venous pressure had been achieved for at least two minutes, the next injection was administered. In group 5 there was no interval between the two injections. Table 1 shows the numbers of microspheres. The number of 15,40 , and 80 $\mu \mathrm{m}$ microspheres per aliquot, used in groups 3 and 4, was approximately 10,25 , and 90 times as many as that of the 15,50 , and $90 \mu \mathrm{m}$ microspheres injected individually in our previous communication. The nominal size of 90 $\mu \mathrm{m}$ diameter microspheres quoted in our previous publication were no longer manufactured by our supplier and we therefore opted for the nearest size range of $80-85 \mu \mathrm{m}$ diameter. The total number of 15 and $80 \mu \mathrm{m}$ microspheres used in groups 1 and 2 represented a seven- and 45-fold increase, respectively, from that used previously. Equivalent volumes of saline were injected into the control group during the same time course. Following completion of the injections, animals were observed for 10-30 minutes until a final steady state portal venous pressure was sustained. Finally, a vascular clamp was applied to the portal vein as close to the liver hilus as possible and the pressures recorded as a measurement of the maximum attainable portal venous pressure. Each experiment was terminated following the completion of each protocol for each respective group and the animals were killed rapidly with a lethal dose of anaesthetic.

\section{MEASUREMENT OF WEDGED HEPATIC VENOUS PRESSURE \\ The measurement of wedged hepatic venous pressure has, until now, only been reported in man and medium sized animals. ${ }^{6}{ }^{17}$ In order to achieve this in the rat, the wedged hepatic venous pressure was measured through a large diameter catheter (outer diameter $1.6 \mathrm{~mm}$ ), with a $30^{\circ}$ bent tip, inserted into the right}


Table 2 Comparison of the changes in portal venous pressure (PVP) and splenic pulp pressure (SPP) in different groups after microsphere injections

\begin{tabular}{|c|c|c|c|c|c|c|c|c|}
\hline \multirow[b]{2}{*}{ Group } & \multicolumn{4}{|l|}{$P V P$} & \multicolumn{4}{|l|}{$S P P$} \\
\hline & Basal & Postinfusion & $\begin{array}{l}\text { Increase } \\
(m m \mathrm{Hg})\end{array}$ & $\%$ increase & Basal & Postinfusion & $\begin{array}{l}\text { Increase } \\
(\mathrm{mm} \mathrm{Hg})\end{array}$ & $\%$ increase \\
\hline 1 & $7.4(1.6)$ & $14.9(3.6)^{\star}$ & $7.5(2.8)^{\star}$ & $102.2(35.6)^{\star}$ & $8.8(2.9)$ & $15.1(3.8)^{\star}$ & $6.3(3.3)^{\star}$ & $90.9(41.8)$ \\
\hline 2 & $6.7(1.4)$ & $24.0(2.2)^{\star}$ & $17.3(2.7)^{\star}$ & $272.3(78.0)^{\star}$ & $10.8(3.2)$ & $22.8(2.5)^{\star}$ & $12.0(4.5)^{\star}$ & $139.3(51.7)$ \\
\hline 3 & $7.7(0.8)$ & $19.9(3.7)$ & $12.3(3.9)$ & $162.8(60.1)$ & $9.3(1.2)$ & $18.7(3.4)$ & $9.3(3.6)$ & $103.1(48.5)$ \\
\hline 4 & $7.4(1.0)$ & $20.3(1.0)$ & $12.9(1.5)$ & $178.6(41.0)$ & $8.7(1.4)$ & $19.8(1.0)$ & $11.2(1.7)$ & $133.5(39.2)$ \\
\hline 5 & $7.9(0.9)$ & $19.0(1.3)$ & $10.9(1.7) \dagger$ & $140.3(32.0) \dagger$ & $10.8(1.4)$ & $18.6(1.4)$ & $7.8(2.1) \dagger$ & $74.9(26.6)^{\star}$ \\
\hline Control & $7.8(0.5)$ & $7.7(0.4)$ & NA & NA & $10.2(1.5)$ & $10.3(1.4)$ & NA & NA \\
\hline
\end{tabular}

${ }^{\star} \mathrm{p}<0.05$ versus other groups,, $\mathrm{p}<0.05$ versus group 3 and 4 , Student's unpaired $t$ test.

jugular vein, advanced through the right atrium, and into a branch of the right anterior hepatic vein.

PARTIAL PORTAL VEIN LIGATED MODEL

Steady state portal venous pressure was compared with the partial portal vein ligated rat. Calibrated portal vein constriction was performed in the control group when the pressures had stabilised for at least 10 minutes following the final injection of saline according to established techniques. ${ }^{8}$

HISTOLOGY

At the termination of each experiment, livers were perfused through the portal vein via a 20 $\mathrm{ml}$ syringe containing a $10 \%$ solution of formaldehyde diluted in phosphate buffered saline at a subphysiological flow rate (less than $10 \mathrm{ml} / \mathrm{min}$ ) to ensure that structural damage was minimal and that tissue fixation was extensive and rapid. Perfusion of the liver was halted when fixation of the liver was deemed complete. This was ascertained by palpation of the perfused liver, the surface of which became hardened on fixation. When perfusion had ceased the livers were excised and stored in containers of fixative. Representative sections were cut at random from each stored liver segment and embedded into wax blocks which then underwent microtomal sectioning and mounting. Sections of lung were also cut from each lobe chosen at random, at the termination of each experiment, and placed immediately

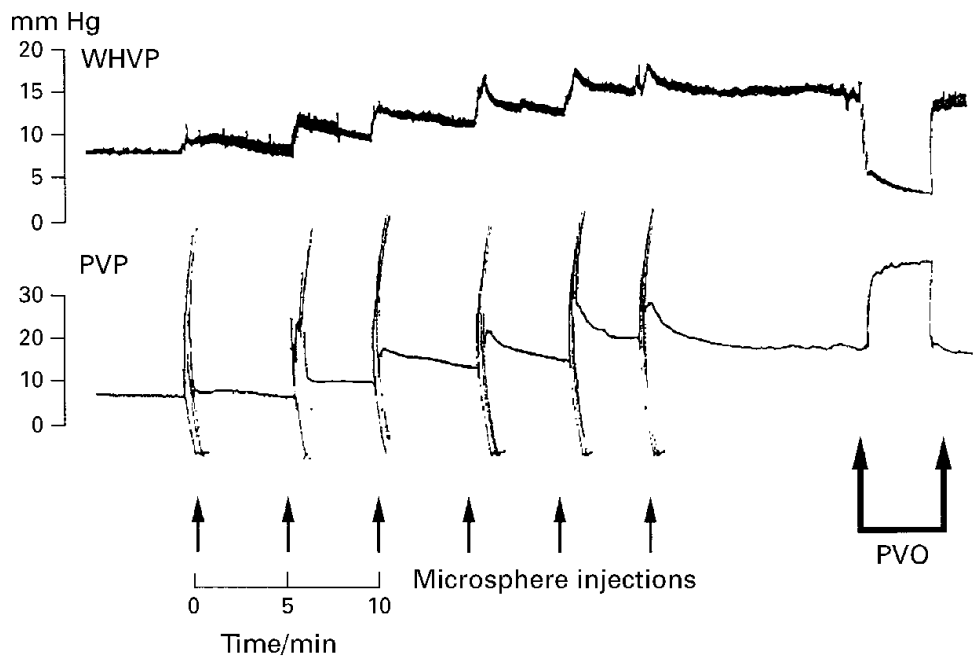

Figure 1 Portal venous pressure (PVP) and wedged hepatic venous pressure (WHVP) recordings from group 1 during intraportal injections of $15 \mu \mathrm{m}$ diameter microspheres and subsequent portal vein occlusion (PVO).



Figure 2 Changes in portal venous pressure (PVP) during intraportal microsphere injections. ${ }^{\star} p<0.05$, ${ }^{\star \star} p<0.01$, Student's unpaired $t$ test, group 2 versus group 1.

into the fixative described above. The mounted sections were then prepared according to the above protocol and all prepared slides were finally stained with haematoxylin and eosin.

STATISTICS

Results are expressed as mean (SD). Statistical comparisons within and between groups were performed using the Student's unpaired $t$ test unless otherwise stated. Results were considered statistically significant at $\mathrm{p}<0.05$.

\section{Results}

PORTAL VENOUS PRESSURE

Basal portal venous pressure was sustained throughout the experimental period in the control group at $7.8(0.5) \mathrm{mm} \mathrm{Hg}$ and was not significantly different to other groups prior to the injection of microspheres ( $p>0.05$, Newman-Keuls analysis of variance, table 2). Injection of $15 \mu \mathrm{m}$ microspheres produced an immediate increase in portal venous pressure which gradually declined to a new steady state within 10 minutes (fig 1). Interestingly, the procedure induced a simultaneous increase in wedged hepatic venous pressure, which also peaked, attained an elevated steady state and was indicative of increased sinusoidal perfusion. Conversely, the wedged hepatic venous pressure decreased following portal venous occlusion and was indicative of decreased sinusoidal perfusion. Two injections of $15 \mu \mathrm{m}$ diameter microspheres (group 1) produced an increase in steady state portal venous pressure of 11.8 (2.2) $\mathrm{mm} \mathrm{Hg}$ (fig 2). Microspheres of 


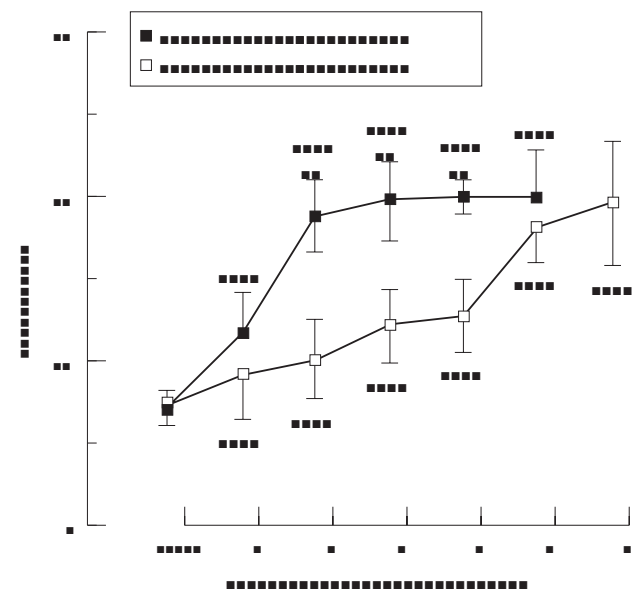

Figure 3 Changes in portal venous pressure (PVP) during intraportal microsphere injections. ${ }^{\star \star} p<0.01$, Student's unpaired t test, group 4 versus group 3.

diameter $80 \mu \mathrm{m}$ (group 2) induced a significant increase in portal venous pressure of 19.4 (4.6) $\mathrm{mm} \mathrm{Hg}$ after two injections and was significantly greater than the final steady state pressure in group $1 \quad(\mathrm{p}<0.05)$. No further significant increases in portal venous pressure were attained following subsequent injections in either group. However, in groups 3 and 4, which received sequential injections of different sized microspheres, a notable increase in portal venous pressure was only observed after injections of the $80 \mu \mathrm{m}$ diameter microspheres (fig 3). In group 5, two sequential bolus injections of $80 \mu \mathrm{m}$ microspheres elicited an immediate increase in the portal venous pressure to 19.0 (1.3) $\mathrm{mm} \mathrm{Hg}$, which was sustained during the observation period of 150 minutes. This was accompanied by an immediate reduction in wedged hepatic venous pressure and systemic blood pressure (fig 4). There were no significant changes in portal venous pressure in the control group after injections of an equivalent volume of saline aliquots (table 2). Splenic
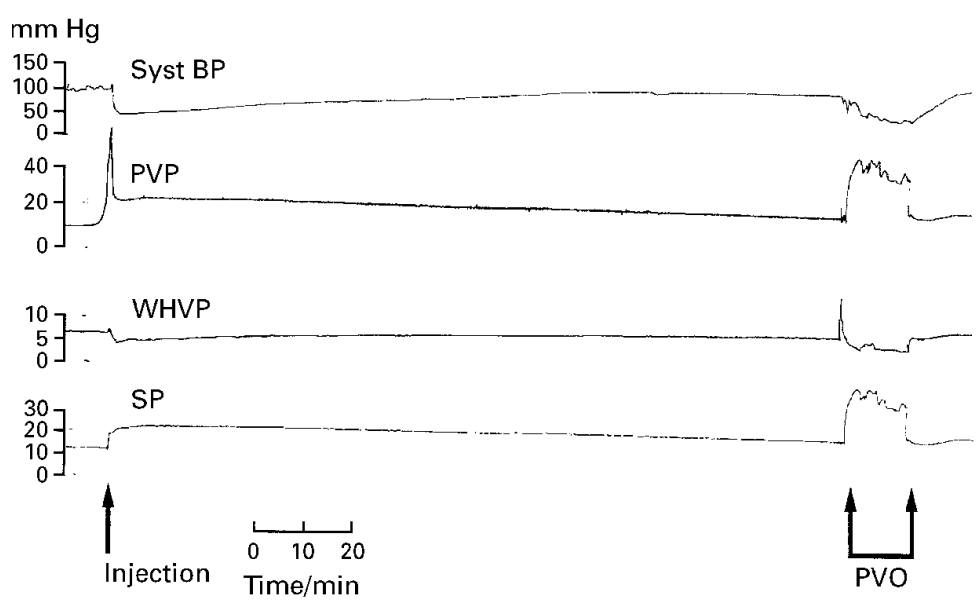

Figure 4 Systemic blood pressure (Syst BP), portal venous pressure (PVP), wedged hepatic venous pressure (WHVP), and splenic pulp pressure (SP) measurements after two aliquots of $80 \mu \mathrm{m}$ diameter microspheres (group 5) were injected continuously into the portal vein. The portal venous pressure was elevated to a steady state value of 19.0 (1.3) mm Hg 150 minutes after injection. However, total occlusion of the portal vein at the liver hilus produced a further dramatic increase in portal venous pressure (PVO) which again suggested that not all the intrahepatic shunts had been occluded. pulp pressure closely paralleled portal venous pressure but was damped to varying degrees.

Portal vein occlusion produced a large, rapid, rise in portal venous pressure (figs 1 and 4) (38.8 (5.7), $36.6(6.0), 37.2(7.5), 41.7$ (6.7), and $43.2(2.4) \mathrm{mm} \mathrm{Hg}$ in groups $1,2,3$, 5 , and control respectively). There was no significant difference between these values ( $p>0.05$, Newman-Keuls analysis of variance between groups). Partial portal vein ligation in control rats (group 6) induced a significant increase in portal venous pressure to 20.7 (0.5) $\mathrm{mm} \mathrm{Hg}$ and was not statistically different to that produced by the two injections of the 80 $\mu \mathrm{m}$ diameter microspheres in group 5 $(p>0.05)$.

WEDGED HEPATIC VENOUS PRESSURE

There was a small but significant difference between the basal wedged hepatic venous pressure and portal venous pressure in all instances where these were measured $(6.9(0.89)$ versus 7.5 (1.1) $\mathrm{mm} \mathrm{Hg}$ respectively, $\mathrm{p}<0.05$, Student's paired $t$ test). Wedged hepatic venous pressure did not change in control rats (group 6) after saline injections, but was significantly reduced by portal venous occlusion and by partial portal vein ligation $(6.7(0.8)$ versus 3.3 $(0.8)$ and $5.4(1.0) \mathrm{mm} \mathrm{Hg}$, respectively, $\mathrm{p}<0.01$, Student's paired $t$ test). Sequential injections of $15 \mu \mathrm{m}$ microspheres in group 1 gradually increased the wedged hepatic venous pressure from a basal value of 7.0 (1.0) $\mathrm{mm} \mathrm{Hg}$ to the final steady state level of $12.4(1.8) \mathrm{mm}$ $\mathrm{Hg}(\mathrm{p}<0.01$, Student's paired $t$ test). This was reduced by portal vein occlusion to $3.0(0.9)$ $\mathrm{mm} \mathrm{Hg}, \mathrm{p}<0.01$ (fig 1 ). In contrast, the wedged hepatic venous pressure was significantly reduced following sequential injections of $80 \mu \mathrm{m}$ microspheres in groups 3 and 4 (6.7 (0.7) versus $3.0(0.6) \mathrm{mm} \mathrm{Hg}, \mathrm{p}<0.01$, Student's paired $t$ test). The two injections of $80 \mu \mathrm{m}$ microspheres in group 5 also caused a significant and immediate decrease in wedged hepatic venous pressure (6.9 (1.0) versus $3.5(0.7) \mathrm{mm} \mathrm{Hg}$, $\mathrm{p}<0.01$ Student's paired $t$ test) but this gradually returned to normal values $(6.7(2.6) \mathrm{mm}$ $\mathrm{Hg}$ ) within 30-40 minutes. This value was reduced by portal venous occlusion to 2.8 (2.0) $\mathrm{mm} \mathrm{Hg}(\mathrm{p}<0.01$, Student's paired $t$ test $)$.

MEAN ARTERIAL PRESSURE

Mean arterial pressure was significantly decreased following the first injection of microspheres in all experimental groups from 97.4 (12.5) to 55.0 (5.1) $\mathrm{mm} \mathrm{Hg} \quad(\mathrm{p}<0.001$, Student's paired $t$ test). Mean arterial pressure in group 5 eventually returned to preinjection values approximately 40 minutes after the two injections of $80 \mu \mathrm{m}$ microspheres (fig 4). No significant change in mean arterial pressure was observed in control rats during saline injections or after partial portal venous ligation (97.4 (12.5) versus 96.7 (14.0) and 95.8 (13.8) mm Hg respectively, $\mathrm{p}>0.05$, Student's paired $t$ test). However portal venous occlusion in control rats produced a significant reduction in mean arterial pressure (96.7 (14.0) versus 51.7 (8.6) $\mathrm{mm} \mathrm{Hg}, \mathrm{p}<0.001$, Student's paired $t$ test) and was similar to that seen after microsphere 

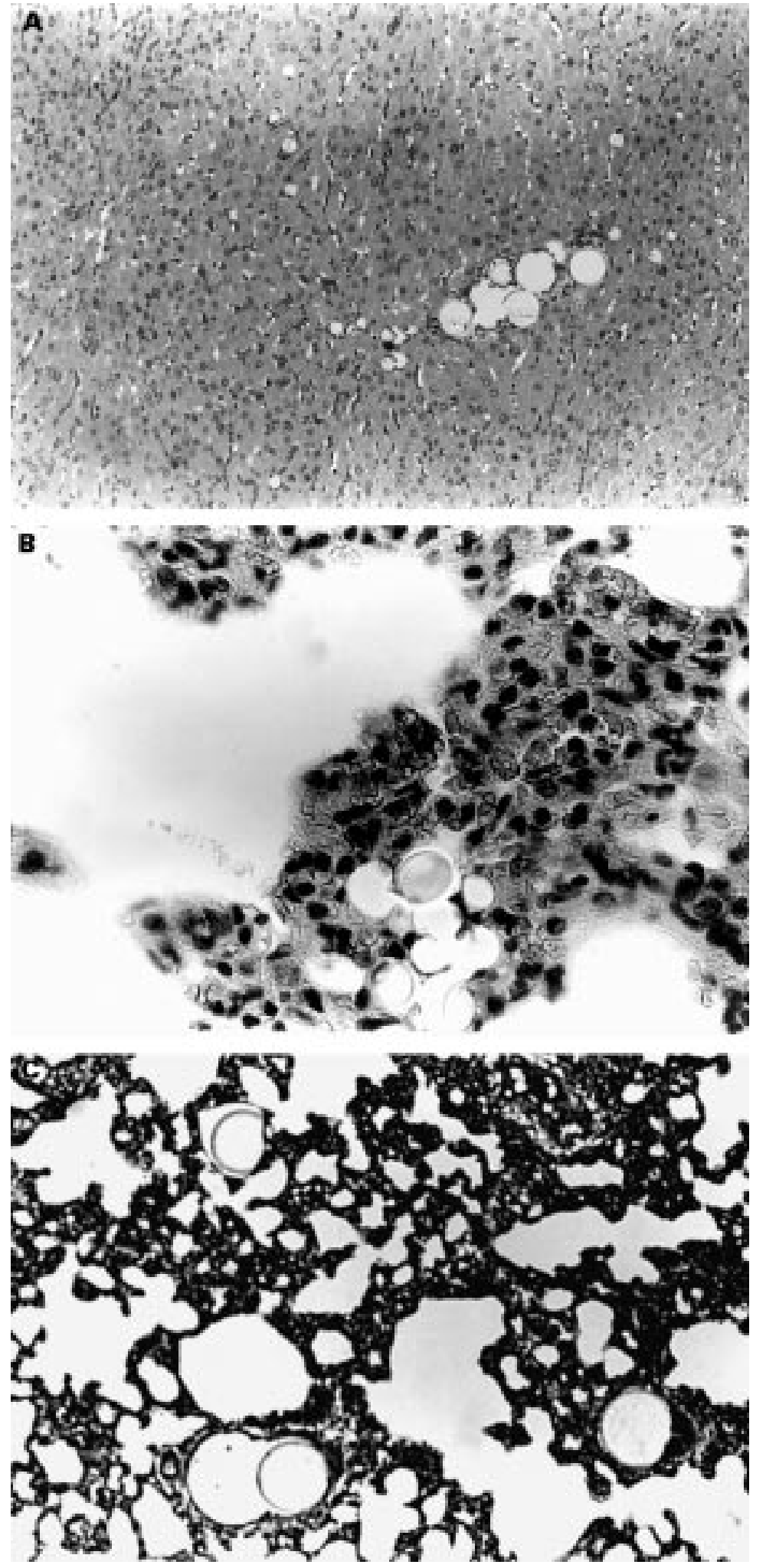

Figure 5 Histological sections showing (A) 15, 40, and 80 um spheres lodged in liver (group 3, original magnification $\times 10$ ), (B) $15 \mu \mathrm{m}$ spheres lodged in lung (group 4, original magnification $\times 40$ ), and $(C) 80 \mu \mathrm{m}$ spheres lodged in lung (group 2 , original magnification $\times 16$ ).

injections (55.1 (5.1) $\mathrm{mm} \mathrm{Hg}$ ) in the experimental groups $(p>0.05)$.

HISTOLOGY

In groups 1 and 2, almost all of the portal radicles observed were blocked by the injected microspheres, with $15 \mu \mathrm{m}$ microspheres (group 1) lodged in the terminal portal venules and 80 $\mu \mathrm{m}$ microspheres (group 2) lodged in the large portal radicles. In group 3, most of the large and small portal radicles were blocked by the different sized microspheres injected sequentially in increasing order of diameter. However, in group 4, portal venules were mainly obstructed by the $80 \mu \mathrm{m}$ spheres, while many $15 \mu \mathrm{m}$ and $40 \mu \mathrm{m}$ spheres injected after the 80 $\mu \mathrm{m}$ diameter spheres were trapped above the $80 \mu \mathrm{m}$ spheres. Results in group 5 were similar to those in group 2, but fewer microspheres were observed in group 2. Microspheres were found in the pulmonary vasculature of all experimental groups. Histological assessment of representative sections from each group, although qualitative, suggested that the lungs of group 2 rats had the largest number of trapped $80 \mu \mathrm{m}$ spheres. Surprisingly, the largest number of $15 \mu \mathrm{m}$ diameter microspheres was found in the lungs of group 4 and not in group 1 animals as expected (fig 5).

\section{Discussion}

The present study showed that the portal venous pressure was increased beyond $50 \%{ }^{12}$ to a range between 100 and $270 \%$ of the basal value by increasing the number of microspheres injected. It appeared that increasing the number injected to $1.5 \times 10^{7} 15 \mu \mathrm{m}$ microspheres or $2.7 \times 10^{5} 80 \mu \mathrm{m}$ microspheres, both administered via three injections, was sufficient to achieve this. The number of microspheres required to generate the maximum portal venous pressure in the present study was determined by two factors: the maintenance of a steady state portal venous pressure which did not increase significantly after three injections, implying that the maximum number of intrahepatic portal radicles had been occluded; and a sustained decrease in mean arterial pressure after three to four microsphere injections, similar to that observed in control rats after total portal vein occlusion, giving rise to extensive mesenteric pooling of portal venous blood.

The present study used a larger number of microspheres than previous studies and clearly indicated that the rise in portal venous pressure correlated with the microsphere diameter. The $80 \mu \mathrm{m}$ microspheres induced a much larger and rapid increase in portal venous pressure compared with $15 \mu \mathrm{m}$ spheres (fig 2). Highly significant differences in mean maximal portal venous pressures of 14.9 (3.6) $\mathrm{mm} \mathrm{Hg}$ and 24.0 (2.2) $\mathrm{mm} \mathrm{Hg}(\mathrm{p}<0.05)$ were induced by sequential injections of either $15 \mu \mathrm{m}$ (group 1) or $80 \mu \mathrm{m}$ (group 2) spheres respectively. These data therefore suggest that intrahepatic shunting occurred through channels that were between $15 \mu \mathrm{m}$ to possibly over $80 \mu \mathrm{m}$ in diameter. However, it was unclear whether this had occurred at the presinusoidal or sinusoidal level, or whether it was intraparenchymal or extraparenchymal shunting, particularly in the absence of quantitative flow data which will be attempted in future studies. Moreover, differentiation between intrahepatic shunt flow from sinusoidal flow might be achieved in future studies by the use of two different isotopes, one 
only being taken up by the parenchyma. However, it is conceivable that if total occlusion of all the intrahepatic portal radicles were possible by microsphere injection, the portal venous pressure produced by any diameter of microspheres would have tended to be similar and approached the value created by portal vein occlusion. Although this was readily achievable in the hepatic arterial vasculature (data not presented) this was not observed in the present study, even though the total number of spheres used in groups 1 and 2 was greatly increased. Maximum occlusion of the intrahepatic portal radicles was confirmed by maximal increases in portal venous pressure, decreased wedged hepatic venous pressure, reduced mean arterial pressure, and unaltered liver histology.

The localisation of $80 \mu \mathrm{m}$ microspheres in the pulmonary vasculature provided additional support for the existence of large intrahepatic portal systemic shunts in the normal rat liver. This hypothesis was further substantiated by the result from the sequential injections of different sized microspheres. Originally it was believed that the regimen of injections used should simultaneously obstruct an extensive range of diameters $(15-80 \mu \mathrm{m})$ of intrahepatic portal radicles, and might therefore induce a greater increase in portal venous pressure than obstruction of only one diameter of shunts using single sized microspheres. This range was selected because the exact sizes of microspheres used originally were no longer available and it was therefore decided to use the nearest sizes obtainable. The only exception, the $15 \mu \mathrm{m}$ diameter microspheres were used in both studies and although a sevenfold increase was used in the number injected in the present study, there were no significant differences in the steady state portal venous pressures between the two separate studies. These two observations suggest that the maximum number of portal venous tracts of this particular diameter (15 $\mu \mathrm{m}$ and $40-50 \mu \mathrm{m})$ were blocked. This was one of the main reasons that this particular study was carried out. The slightly smaller cannulae used in the first study did not permit larger numbers of $90 \mu \mathrm{m}$ diameter microspheres to be injected per aliquot due to blockage problems and it was essential to use identical numbers of aliquots between the various groups in order to eliminate haemodynamic variability due to differences in systemic blood volume. Thus the limitations in the number of aliquots injected and the much smaller total number of $90 \mu \mathrm{m}$ diameter microspheres injected $\left(1 \times 10^{4}\right.$ compared with $1.8 \times 10^{5}$ of 80 $\mu \mathrm{m}$ diameter microspheres in the present study) may be why steady state portal venous pressures were much smaller in the earlier study. However, the present result obtained was unexpected using an assortment of microspheres of differing diameters; the final portal venous pressure obtained was similar to that produced by two injections of $80 \mu \mathrm{m}$ microspheres (fig 3). This suggested that the final portal venous pressure was dependent on the effect of the largest spheres. This presumably was because the larger microspheres obstructed the portal radicles at a higher anatomical level than the smaller microspheres and therefore the effect of the smaller microspheres was obscured. The main reason that such a dramatic increase in steady state portal venous pressure was attained during the present study was because larger numbers of microspheres could be injected due to the slightly larger diameter of cannula used. The fact that further increases in portal venous pressure could not be induced by further increasing the number of microspheres injected is therefore further evidence that the maximum number of portal venous tracts of this particular size $(80 \mu \mathrm{m})$ were blocked. Nonetheless the possibility existed that more portal venous tracts could have been occluded if larger sized microspheres had been used but this was not possible because of the small internal diameter of the cannula used.

The lack of further increases in portal venous pressure could only be attributed to the presence of patent portal venous tracts (less than $80 \mu \mathrm{m}$ in diameter) or induction of the opening of patent intrahepatic portal systemic tracts. The existence of intrahepatic portal systemic shunts was further supported by the measurement of wedged hepatic venous pressure performed using a modification of an established technique. ${ }^{17}$ The wedged hepatic venous pressure reflected the sinusoidal pressure and, because of the extremely low presinusoidal resistance, approximated the portal venous pressure. A reduced portal (and arterial) flow through the sinusoids would therefore be expected to decrease the wedged hepatic venous pressure, and this was observed during extrahepatic portal venous occlusion in the present study. Theoretically, this should also have been observed following complete presinusoidal blockage of the intrahepatic portal radicles. However, the data implied that the changes in wedged hepatic venous pressure were dependent on microsphere diameter and this may have been due to the existence of patent intrahepatic shunts. It is suggested that when the intrahepatic portal radicles were blocked, the raised portal venous pressure induced intrahepatic shunts to open above the level of obstruction. Portal blood then flowed through these shunts, entered the hepatic veins, and therefore increased the wedged hepatic venous pressure. Therefore the degree of shunting, in terms of the percentage of the total hepatic blood flow, was correlated to the resting portal venous pressure and the change in wedged hepatic venous pressure and accounted for differences observed between the small and large diameter microsphere injections.

The synchronous increase in wedged hepatic venous pressure which accompanied the increased portal venous pressure following injection of $15 \mu \mathrm{m}$ microspheres (fig 1) suggested that patent intrahepatic shunts were induced to open acutely to result in this increase. Alternatively, this may have been due to induction of hepatic arterial hyperaemia, in response to reductions in portal venous flow ${ }^{18}$ initiated by microsphere injections, although this does not adequately explain the decreased wedged 
hepatic venous pressure following injection of $80 \mu \mathrm{m}$ diameter microspheres. The decrease in wedged hepatic venous pressure after $80 \mu \mathrm{m}$ microsphere injections may have been due to: (1) blockage of a larger proportion of the portal venous vasculature due to the high anatomical level where occlusion was achieved; (2) blockage of the portal venous vasculature at an anatomical level above the location of putative large (greater than $25 \mu \mathrm{m}$ ) intrahepatic portal systemic shunts; or (3) the existence of fewer large shunts (diameter greater than $80 \mu \mathrm{m}$ ). This has been reported during cirrhosis in man where it was suggested that the frequency of large shunts (diameter greater than $25 \mu \mathrm{m}$ ) was too low to result in a reduced (less than $20 \%$ ) intrahepatic portohepatic shunt index. ${ }^{7}$ However, blockage of portal venous tracts by microsphere injections in vitro, induced a dramatic reduction in hepatic parenchymal glucose uptake that could not be attributed to increased regional portal venous blood flow redistribution via unblocked sinusoids. ${ }^{1920}$ Therefore, the more likely passage of microspheres through the liver, particularly of the 80 $\mu \mathrm{m}$ diameter spheres, into the pulmonary vasculature was through extrahepatic parenchymal routes.

It is concluded from the results of groups $1-4$, that a sustained increase in portal venous pressure with the recovery of the mean arterial pressure can be induced following two injections $\left(1.8 \times 10^{5}\right)$ of $80 \mu \mathrm{m}$ diameter microspheres. This was confirmed in group 5 which showed a normal mean arterial pressure of 99.2 (23.8) $\mathrm{mm} \mathrm{Hg}$ and increased portal venous pressure of 19.0 (1.3) $\mathrm{mm} \mathrm{Hg}$. This value of portal venous pressure was comparable to the mean of $20.7 \mathrm{~mm} \mathrm{Hg}$ found in portal vein ligated rats. ${ }^{36}$ In comparison with the portal vein ligated model of chronic portal hypertension, this microsphere model is acute, intrahepatic, and characterised by the opening of intrahepatic portal systemic shunts. These may have a protective pathophysiological role when the liver is subjected to acute episodes of portal hypertension such as severe viral hepatitis or from liver damage induced by paracetamol overdose.
1 Fernandez-Munoz D, Caramelo C, Santos JC, Blanchart A, Hernando L, Lopez-Novoa JM. Systemic and splanchnic haemodynamic disturbances in conscious rats with experimental liver cirrhosis without ascites. Am $f$ Physiol 1985;249:G316-20.

2 Vorobioff J, Bredfeldt JE, Groszmann RJ. Increased blood flow through the portal system in cirrhotic rats. Gastroenterology 1984;87:1120-6.

3 Geraghty JG, Angerson WJ, Carter DC. Portal venous pressure and portal-systemic shunting in experimental portal hypertension. Am f Physiol 1984;257:G52-7.

4 Bosch J, Mastai R, Kravetz D, Navasa M, Rhodes J. Hemodynamic evaluation of the patient with portal hypertension. Semin Liver Dis 1986;6:309-17.

5 Braillon A, Cailmail S, Gaudin C, Lebrec D. Reduced splanchnic vasoconstriction to angiotensin II in conscious rats with biliary cirrhosis. F Hepatol 1993;17:86-90.

6 Groszmann RJ, Glickman M, Blei AT, Storer E, Conn HO. Wedged and free hepatic venous pressure measured with a balloon catheter. Gastroenterology 1979;76:253-8.

7 Hoefs JC, Reynolds TB, Pare P. A new method for the measurement of intrahepatic shunts. F Lab Clin Med 1984; 103:446-61.

8 Myking AO, Halvorsen JF. A method for graded portal vein stenosis in rats: survival related to degree of stenosis. Eur Surg Res 1973;5:454-7.

9 Tamayo RP. Is cirrhosis of the liver experimentally produced by $\mathrm{CCl}_{4}$ an adequate model of human cirrhosis? Hepatology 1983;3:112-20

10 Sieber CC, Lopez-Talavera JC, Groszmann RJ. Role of nitric oxide in the in vitro splanchnic vascular hyporeactivty in ascitic cirrhotic rats. Gastroenterology 1993;104:17504.

11 Lee SS, Girod C, Braillon A, Hadengue A, Lebrec D. Hemodynamic characterisation of chronic bile duct-ligated G176-80.

12 Jaffe V, Alexander B, Mathie RT. Intrahepatic portal occlusion by microspheres: a new model of portal hypertension in the rat. Gut 1994;35:815-18

13 Wood AJJ, Villeneuve JP, Branch RA, Rogers LM, Shand DG. Intact hepatocyte theory of impaired drug metabolism in experimental cirrhosis in the rat. Gastroenterology 1979;76:1358-62.

14 Okuda K, Suzuki K, Musha H, Arimizu N. Percutaneous transhepatic catheterisation of the portal vein for the study of portal haemodynamics and shunts. Gastroenterology 1977;73:279-84.

15 Huet P-M, Gilles P-L, Villeneuve J-P, Varin F, Viallet A. Intrahepatic circulation in liver disease. Semin Liver Dis 1986;6:277-86.

16 Lin HC, Yu PC, Lee SD, Tsai YT, Kuo JS, Yany MCM. Effects of reserpine administration in two models of portal hypertension in rats. F Hepatol 1993;19:413-7.

17 Lautt WW, Legare DJ. Passive autoregulation of portal venous pressure: distensible hepatic resistance. $A m \mathcal{f}$ Physiol 1992;263:G702-8.

18 Lautt WW. Mechanism and role of intrinsic regulation of hepatic arterial blood flow: the hepatic arterial buffer response. Am f Physiol 1985;249:G549-56.

19 Alexander B, Cottam H, Naftalin RJ. Evidence that rat hepatic arterial pressure and altered pre-sinusoidal resistance affect intrahepatic shunt flow in vitro. F Physiol 1996; 495:102.

20 Alexander B, Naftalin RJ, Rogers C. Evidence from in vitro glucose uptake that hepatic arterial perfusion is required to
maintain the sinusoids open to portal venous perfusion. $\mathcal{F}$ Physiol 1996;495:97. 